\title{
Correlation between Resilience and Mental Health with Lifestyle and Parenting Styles of Veterans' Spouses A Case Study of Ardakan City
}

\section{ART I C L E INF O}

\section{Article Type}

Descriptive Study

\section{Authors}

Abedini Baltork M.*1 $P h D$

Mir Shamsi F.S. ${ }^{2} M S C$

How to cite this article
Abedini Baltork M, Mir Shamsi F.S.
Correlation between Resilience
and Mental Health with Lifestyle
and Parenting Styles of Veterans'
Spouses A Case Study of Ardakan
City. Iranian Journal of War \& Pub-
lic Health.2019;11(3):161-167.

\section{A B S T R A C T}

Aim(s): The physical and mental problems that exist among the veterans have the greatest impact on their spouses, which affects the whole family. The aim of the present study was to investigate the correlation between lifestyle and parenting style with resiliency and mental health of veterans' spouses.

Instruments \& Methods: This descriptive-correlational study was carried out on 170 people of veterans' spouses in Ardakan city in 2018. The subjects were selected by convenience sampling method. Data were collected by the Miller-Smith lifestyle assessment inventory, Baumrind's parenting style inventory, Connor-Davidson resilience scale, and Goldberg and Hillier health questionnaire. Data were analyzed through SPSS 21 software using Pearson correlation coefficient method.

Findings: There was a significant correlation $(\mathrm{p}=0.001)$ between the lifestyle with resiliency $(\mathrm{r}=0.559)$ and mental health $(\mathrm{r}=-0.277)$. Also authoritarian parenting style had a negative significant correlation $(\mathrm{r}=-0.125 ; \mathrm{p}=0.042)$ with resilience, and positive significant correlation $(\mathrm{r}=0.094 ; \mathrm{p}=0.048)$ with mental health. The correlation between powerful parenting style and mental health was negative and significant $(r=-0.133 ; p=0.024)$.

Conclusion: There is correlation between lifestyle and parenting style with resiliency and mental health of veterans' spouses.

Keywords Life Style; Parenting Styles; Resilience; Mental Health; Spouses; Veterans
${ }^{1}$ Education Department, Humanities \& Social Sciences Faculty, University of Mazandaran, Babolsar, Iran ${ }^{2}$ Education Department, Humanities Faculty, Ardakan Branch, Islamic Azad University, Yazd, Iran

\section{*Correspondence}

Address: Education Department, Humanities \& Social Sciences Faculty, University of Mazandaran, Babolsar, Iran

Phone: +98 (11) 35303000

Fax: +98 (11) 3532601

abedini.gilan@gmail.com

\section{Article History}

Received: December 11, 2018

Accepted: June 16, 2019

ePublished: July 21, 2019

\section{T A T I O N LINKS}

[1] The effect of training conflict resolution ... [2] Directory services to veterans and their ... [3] The effect of spiritual components training on ... [4] comparison between mental health of devotee's ... [5] Investigation of personality traits of wives of spinal cord ... [6] Growth Psychology ... [7] Relationship between spiritual intelligence, hardiness and ... [8] Kaplan \& Sadock's synopsis of psychiatry behavioral ... [9] The relationship between emotional intelligence and ... [10] Quality of life, vulnerability and resilience: a qualitative study ... [11] Mental health consequences of war: a brief review of ... [12] Veteran women: mental health-related consequences ... [13] Depression, anxiety, and stress in partners ... [14] Psychological and behavioral problems in children ... [15] Psychosocial outcomes and predictors of distress ... [16] Levels of distress among women veterans attending ... [17] Prevalence of past year substance use and mental ... [18] Religious beliefs and lifestyle with quality of life of ... [19] Family mental ... [20] Theories and application of psychological ... [21] The relationship between parenting styles, shyness ... [22] The relation of attachment styles, emotion ... [23] Psychometric analysis and refinement of the Conner ... [24] Internal consistency and confirmatory factor ... [25] Assessment of the validity and reliability of ... [26] Reliability, validity and factor structure of the ... [27] Relationship between parenting and resilience ... [28] The effect of training Forgiveness on Resilience ... [29] The effect of parenting styles on self-efficacy and mental ... [30] The effectiveness of mindfulness-based ... [31] The effects of parenting styles on students ... [32] Parenting style and social anxiety among ... [33] The relationships between perceived parental ... [34] The relationship between parenting styles ... [35] Parenting style, resilience, and mental health ... [36] The relationship between parenting ... [37] Association between social phobia and ... [38] Psychosocial consequences of ... [39] Influence of parenting style on children's ... 
اعضاى خانواده جانباز بهخصوص همسر وى كه با عوامل فشارزاى مخاى

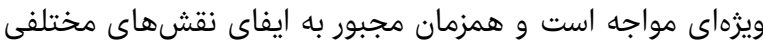

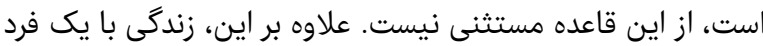

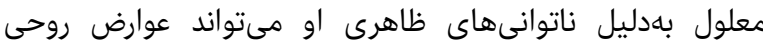

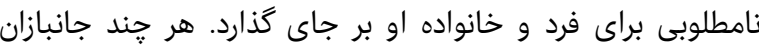

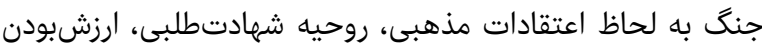

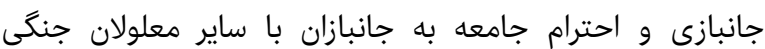

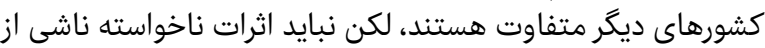

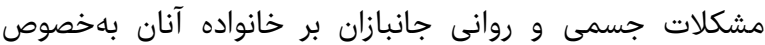

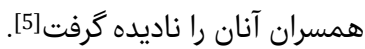

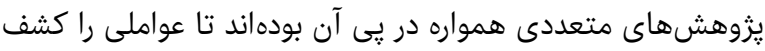

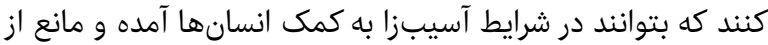

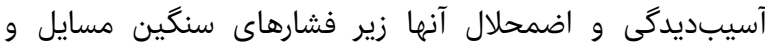

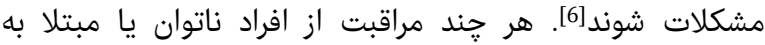

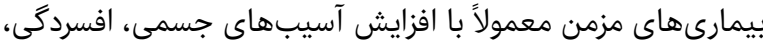

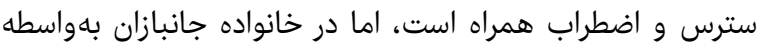

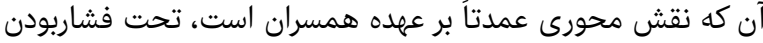

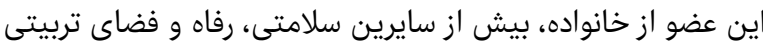

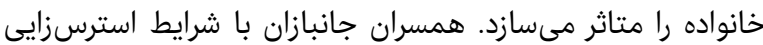

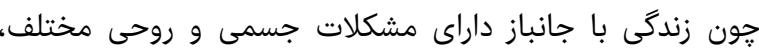

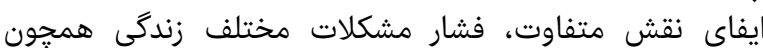

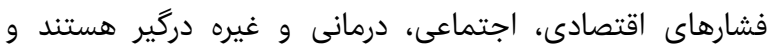

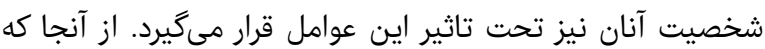

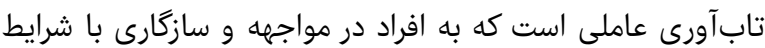

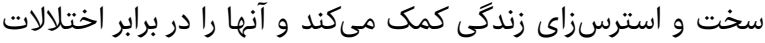

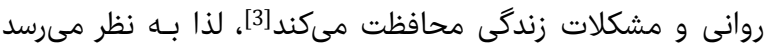

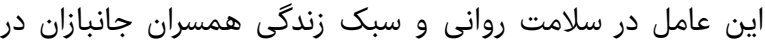

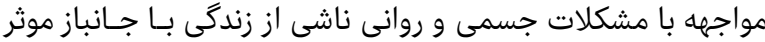

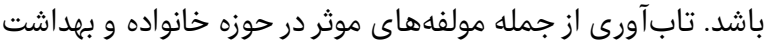

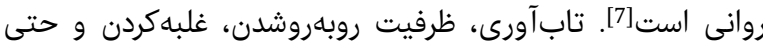

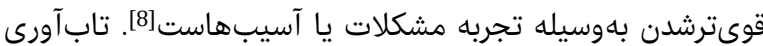

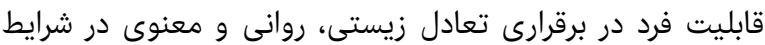

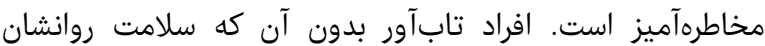
كاهش يابد يا دحار بيمارى شوند، رويدادهاى فشارئ آنارزا را يشت سر سران

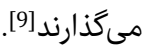

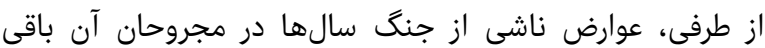

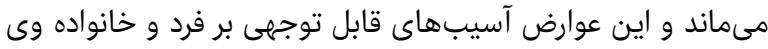

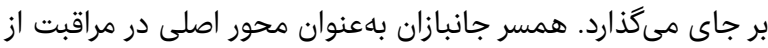

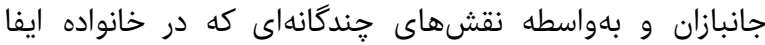

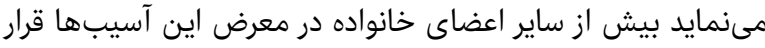

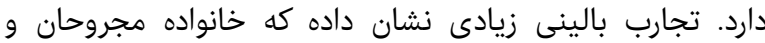

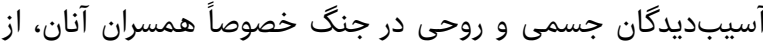

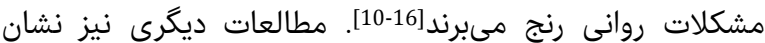

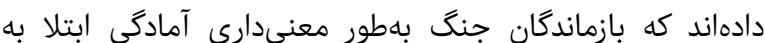

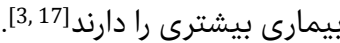

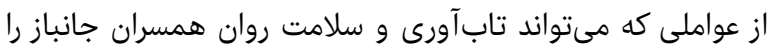

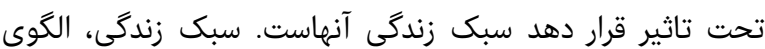

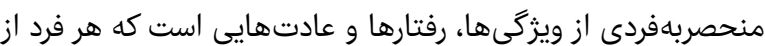

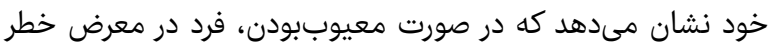

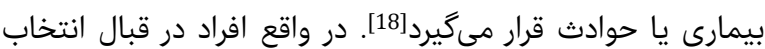

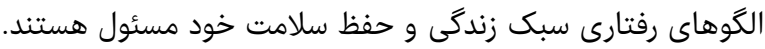
بسيارى از بيمارىها امروزه در اغلب كشورهاى در حال سلام توسعه با

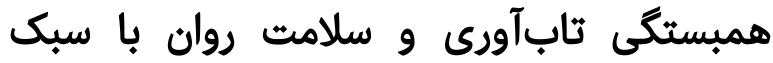
زندگى و سبكهاى فرزنديرورى همسران جانبازان مطالعه موردى شهرستان اردكان فروندئ

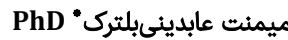
كروه علوم تربيتى، دانشكده علوم انسانى و اجتماعى، دانشكاه مازندران، بابلسر، ايران

فاطمهسادات ميرشمسى دانى

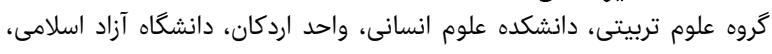
يزد، ايران

جكيده

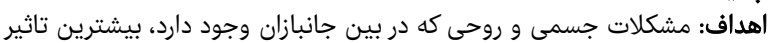

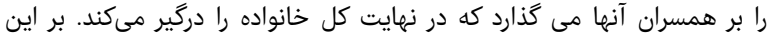

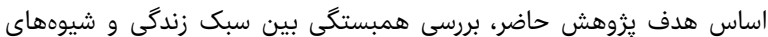

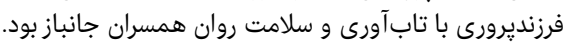

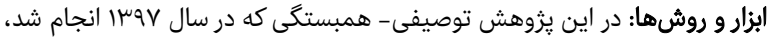

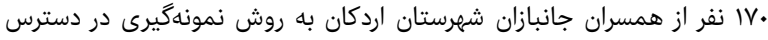

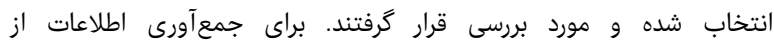

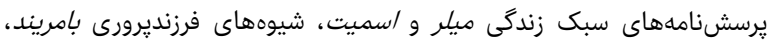

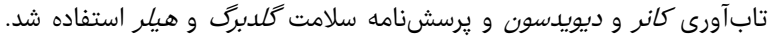

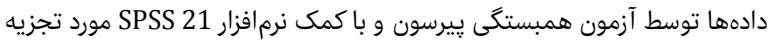
و ت تحليل قرار كرفتند.

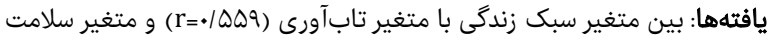

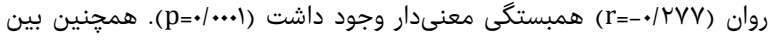

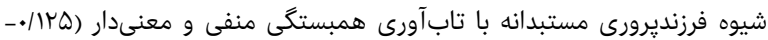

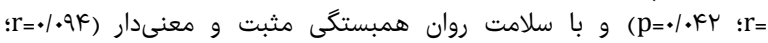

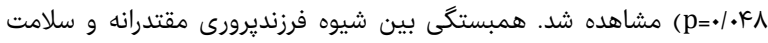

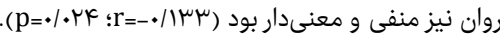

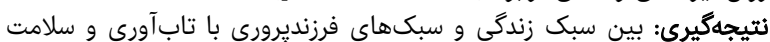

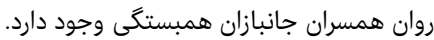
كليدوازمها: سبك زندگى، سبك فرزنديرورى، تابآورى، سلامت روان، همسران، جانبازان

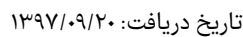

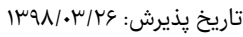

"نويسنده مسئول: abedini.gilan@gmail.com

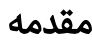

جنغ بهعنوان يكى عامل فشارزاى روانى، ييامدهاى گَستردهاى را در

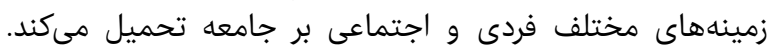

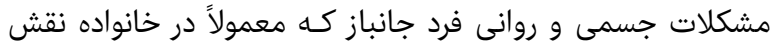

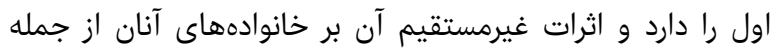

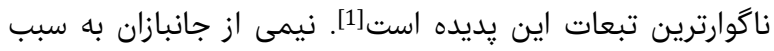

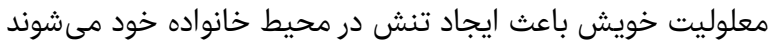

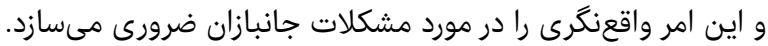

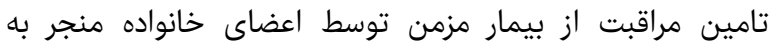

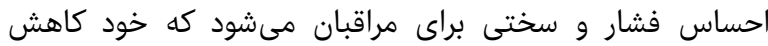

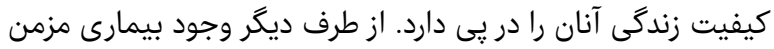

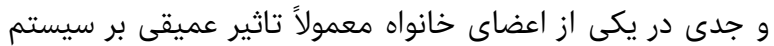

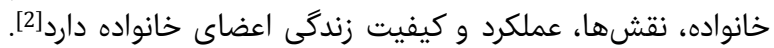

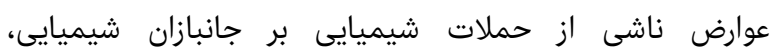

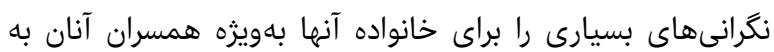

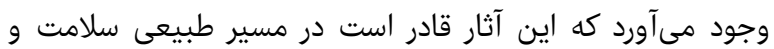

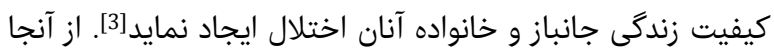

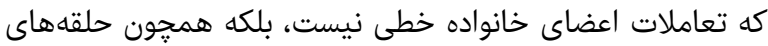
زنجير به هم وابسته بوده و بر يكديكر تاثير متقابل دارد[4]، لذان 


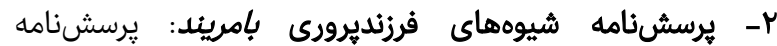

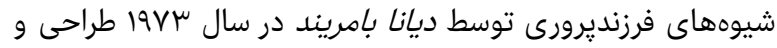

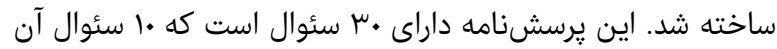

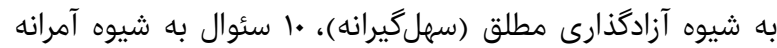

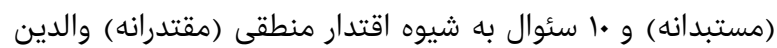

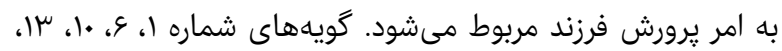

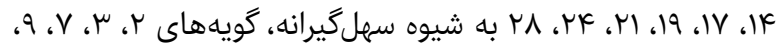

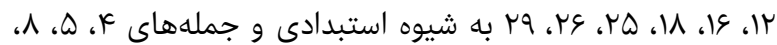

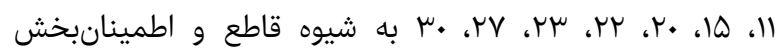

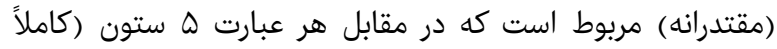

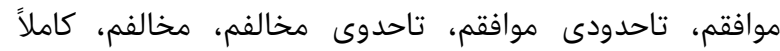

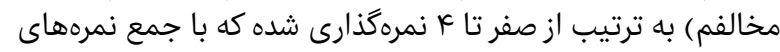

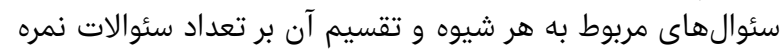

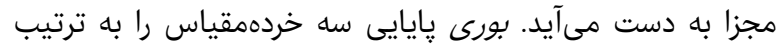

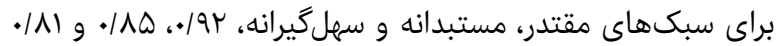

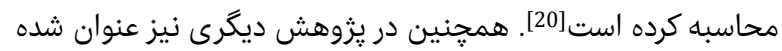

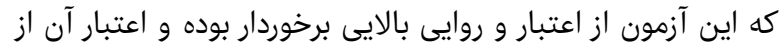

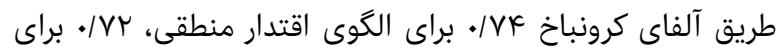

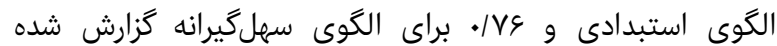

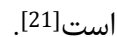
س- هرسشنامه تابآورى كانر و ديويدسون: اين يرسشنامه كه در

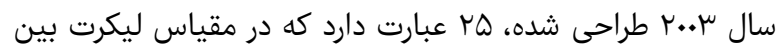

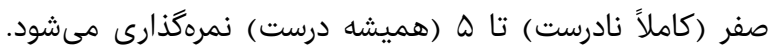

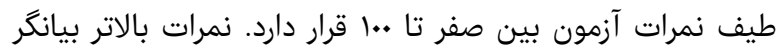

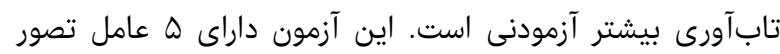

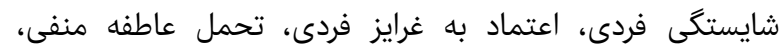

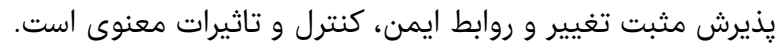

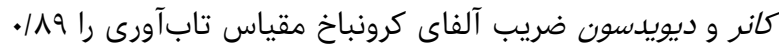

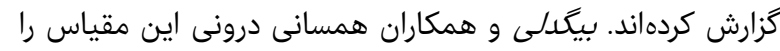

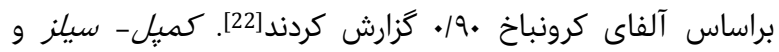

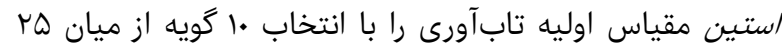

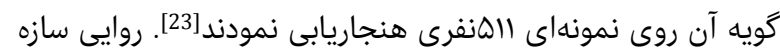

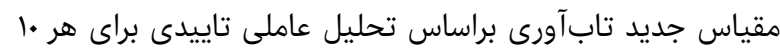

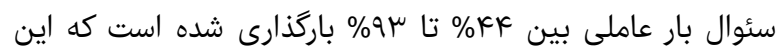

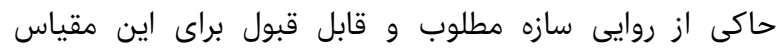

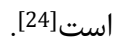

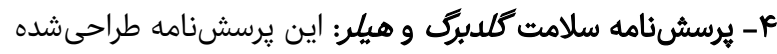

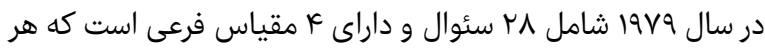

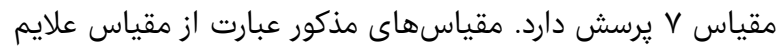

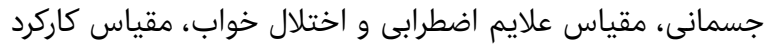

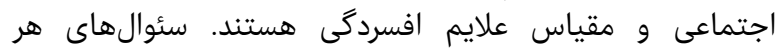

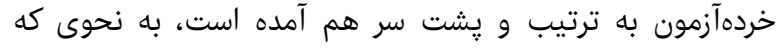

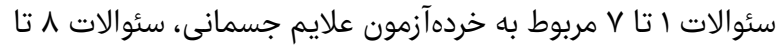

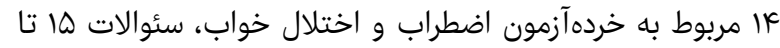

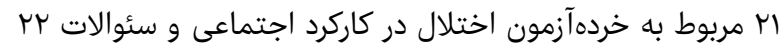

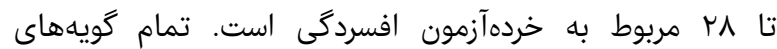

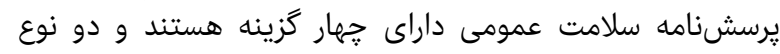

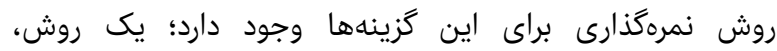

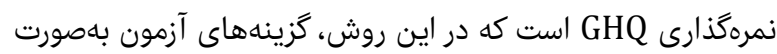

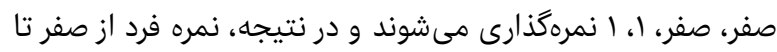

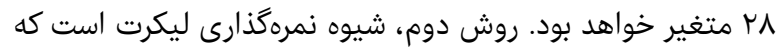

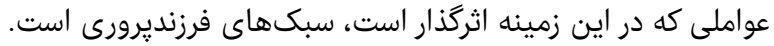

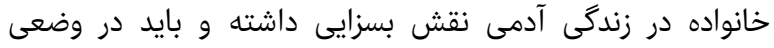

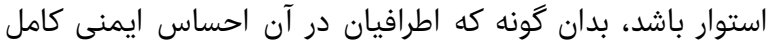

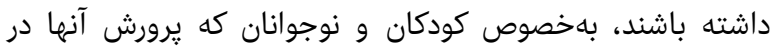

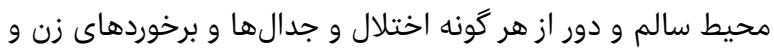

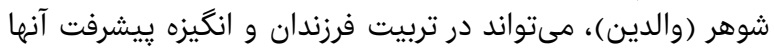

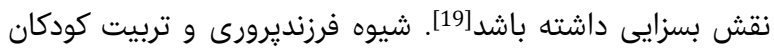

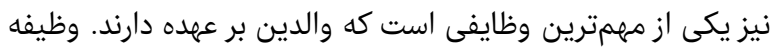

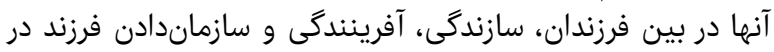

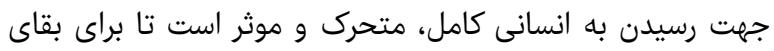

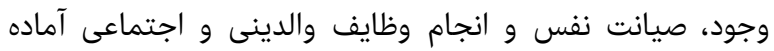

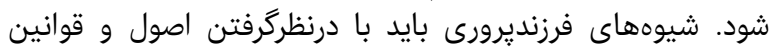

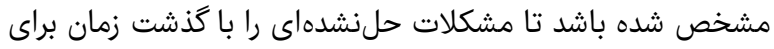

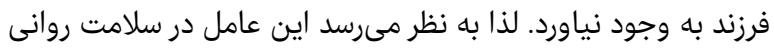

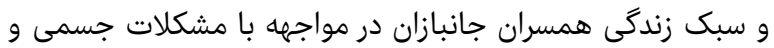
روانى ناشى از زندگى با جانباز موثر باشد جاند.

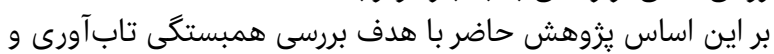

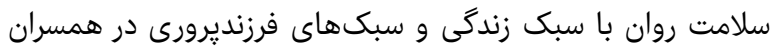

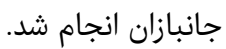

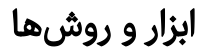

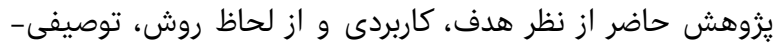

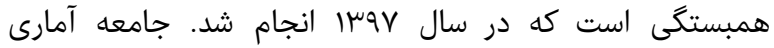
يزوهش، كليه همسران جانبازان شهرستان اردكان (حدود ..ب نفر)

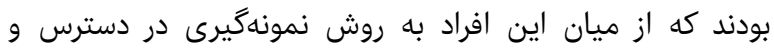

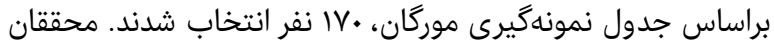

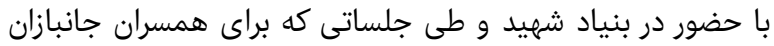

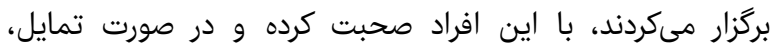

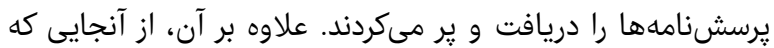

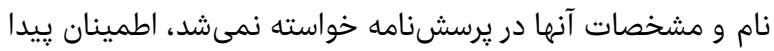

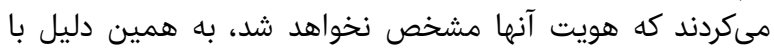

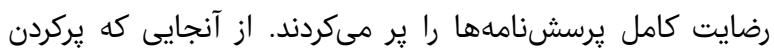

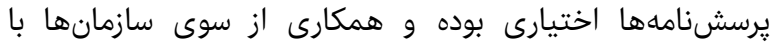

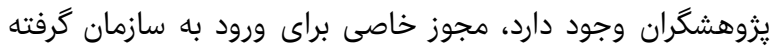

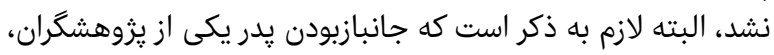

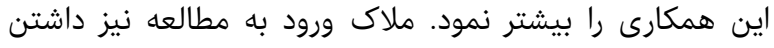

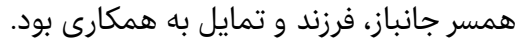

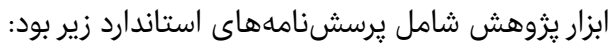

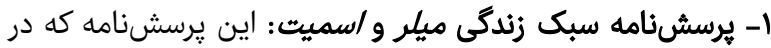

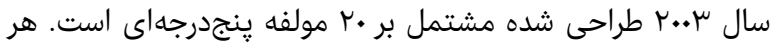

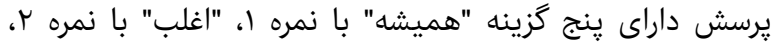

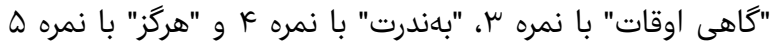

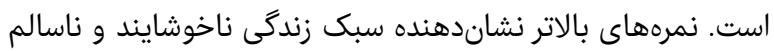

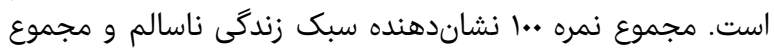

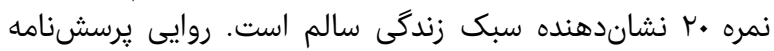

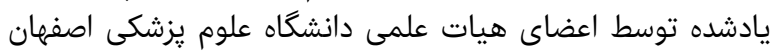

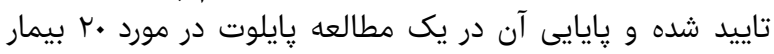

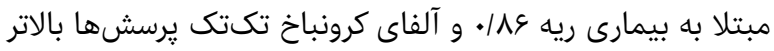

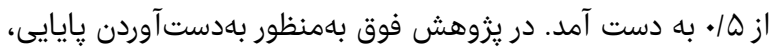

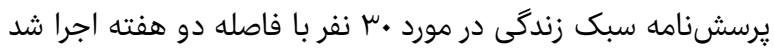

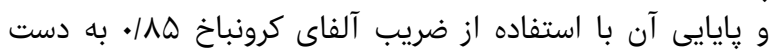


جدول 1) ميانگين آمارى نمرات متغيرهاى سبك زندگىى، تابآورى و و

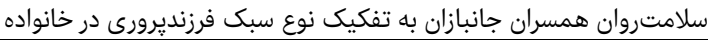

\begin{tabular}{|c|c|}
\hline نمرات & متغيرها \\
\hline & سبك زندگى \\
\hline$V \Delta / \wedge \Delta \pm q / r \Delta$ & شيوه سهلگيرانه \\
\hline 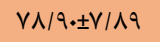 & شيوه مستبدانه \\
\hline$V K / \& 9 \pm 9 / F \Delta$ & شيوه مقتدرانه \\
\hline & تابآورى \\
\hline$\Delta \cdot / 4 \wedge \pm r \cdot / r V$ & شيوه سهلكيرانه \\
\hline$\Delta Q / \vee \backslash \pm 19 / \wedge \varsigma$ & شيوه مستبدانه \\
\hline$\Delta 1 / \cdot \varphi \pm r \cdot / 9$. & شيوه مقتدرانه \\
\hline & سلامت رولن \\
\hline$\mu \Delta / \Lambda \Delta \pm I \kappa / \wedge q$ & شيوه سهلگيرانه \\
\hline$\mu / / 1 q \pm q / / r$ & شيوه مستبدانه \\
\hline$\mu س / 4 . \pm|r / q|$ & شيوه مقتدرانه \\
\hline
\end{tabular}

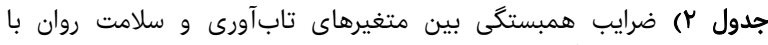

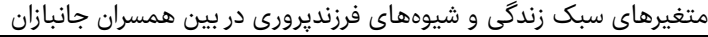

\begin{tabular}{|c|c|c|c|c|}
\hline \multicolumn{2}{|c|}{ سلامت روان } & \multicolumn{2}{|c|}{ تابآورى } & \multirow{3}{*}{ متغيرها } \\
\hline سطح & ضريب & سطح & ضريب & \\
\hline معنىدارى & همبستخى & معنىدارى & همبستگى & \\
\hline$\cdot / \cdots 1$ &.$- / T V V$ & $\cdot / \cdots 1$ & $\cdot / \Delta Q Q$ & سبك زندگى \\
\hline 每 & $\cdot / \cdot \wedge 9$ & •/IV^ & $-* \cdot v 1$ & شيوه فرزنديرورى سهل گيرانه \\
\hline $.1 . k \Lambda$ & $.1 .9 k$ & $.1 . k r$ &.$- / 1 T \Delta$ & شيوه فرزنديرورى مستبدانه \\
\hline $.1 . Y F$ & - & .1 .9$. &.$/ 119$ & شيوه فرزنديرورى مقتدرانه \\
\hline
\end{tabular}

بحث

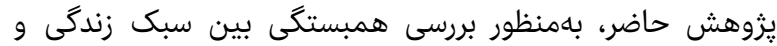

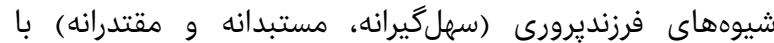

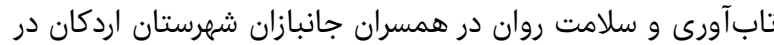

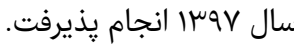

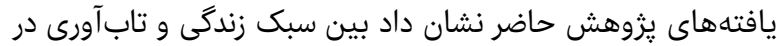

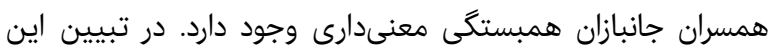

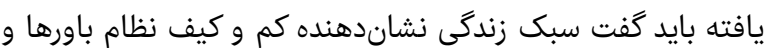

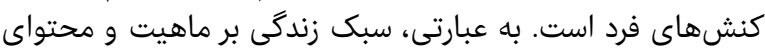

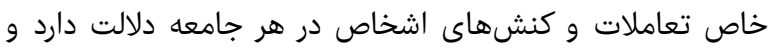

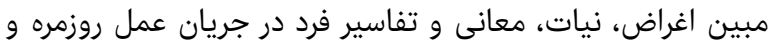

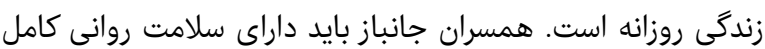

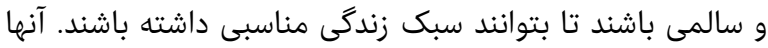

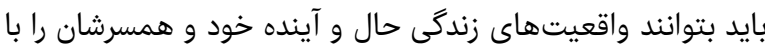

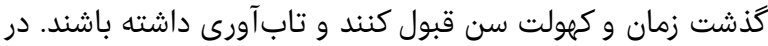

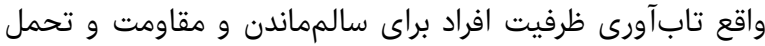

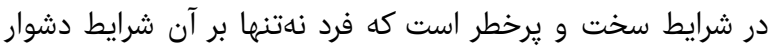

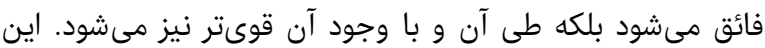

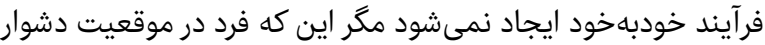

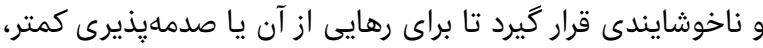

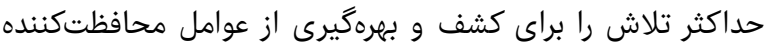

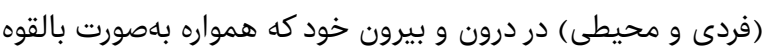
وجود دارد به كار گيرد.

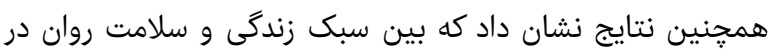

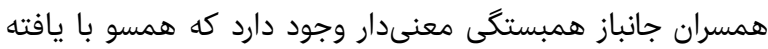

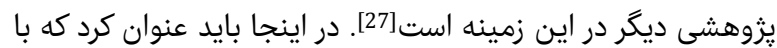

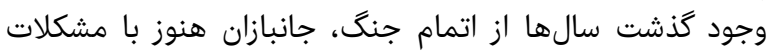

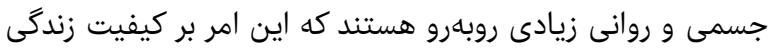

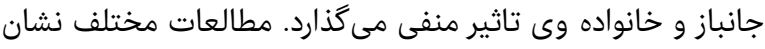

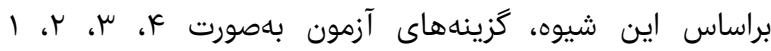

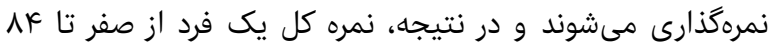

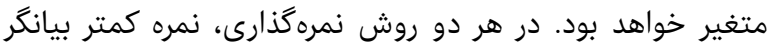

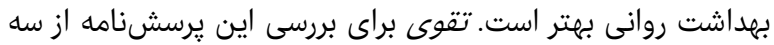

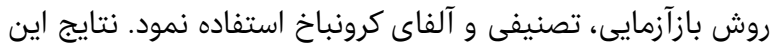

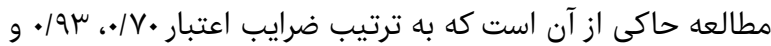

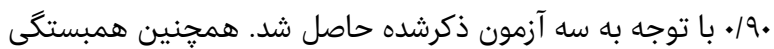

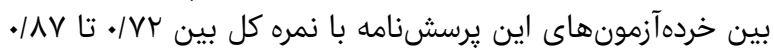

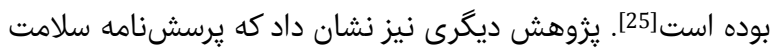

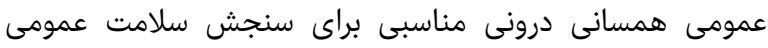

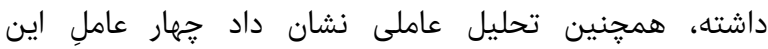

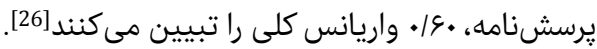

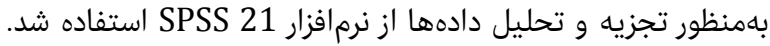

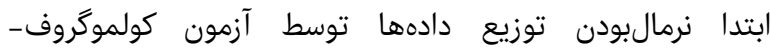

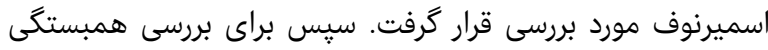

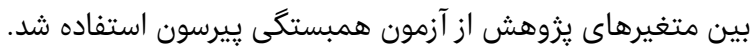

يافتهها از •lV نمونه مورد مطالعه،

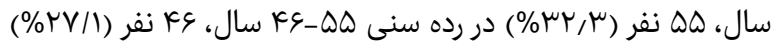
در رده سنى

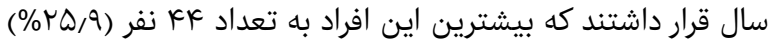

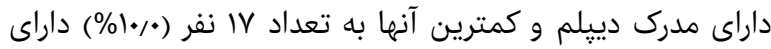
مدرك كارشناسى ارشد بودند.

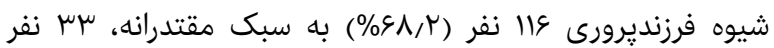

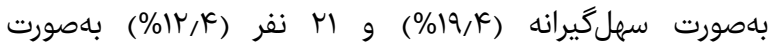

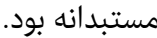

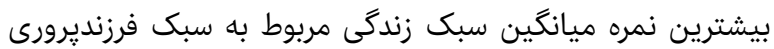

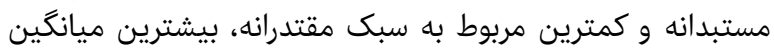

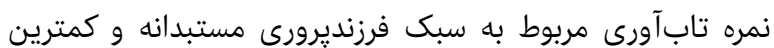

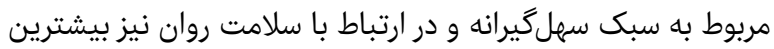

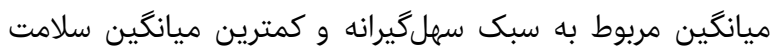

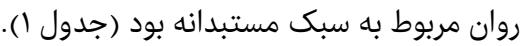

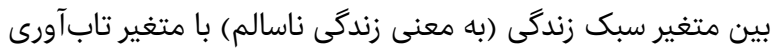

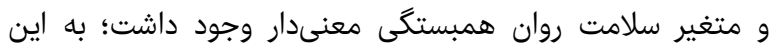

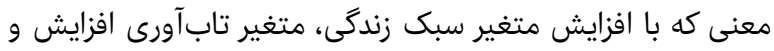

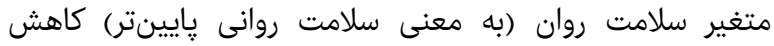

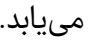
بين شيوه فرزنديرورى مستبدانه با تابآورى همبستگى منفى و معنىدار وجود داشت، بدان معنى كه با افزايش استفاده از اين سبك،

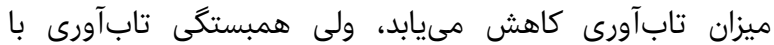

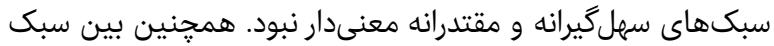

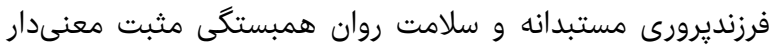

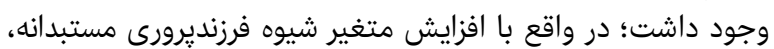

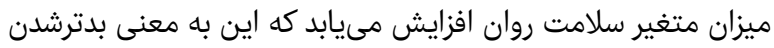

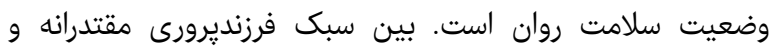

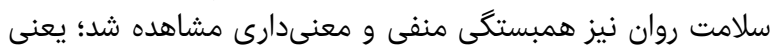

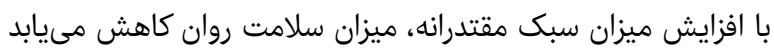

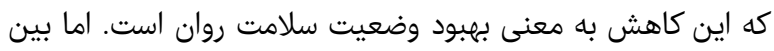

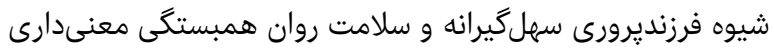

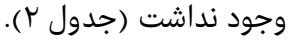




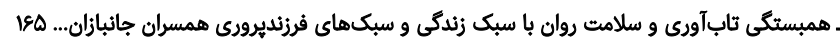

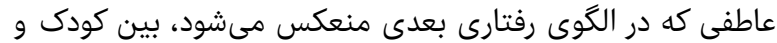

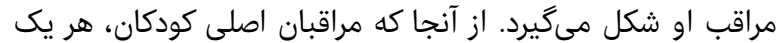

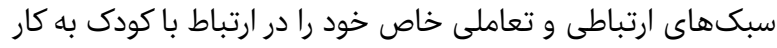

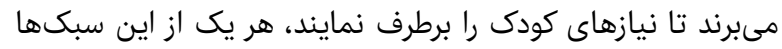

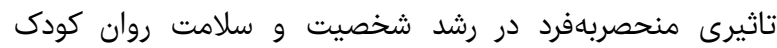

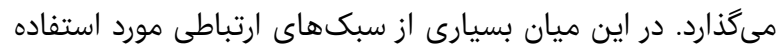

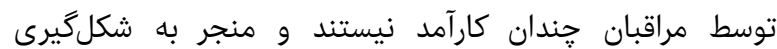

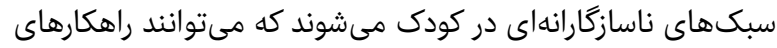

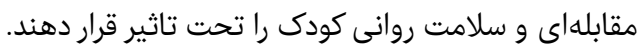

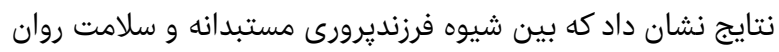

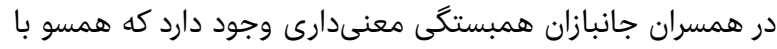

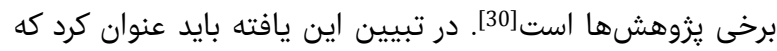

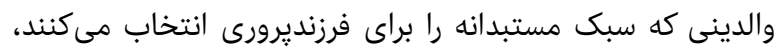

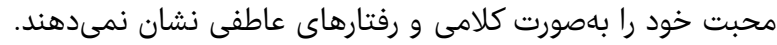

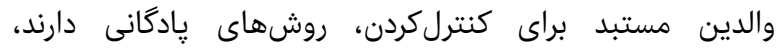

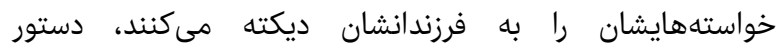

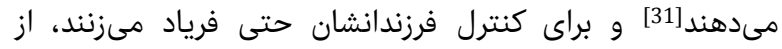

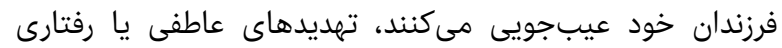

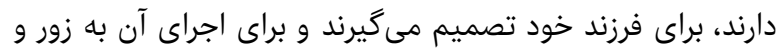

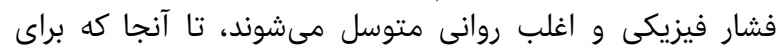

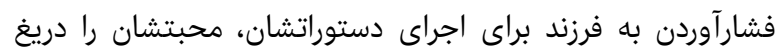

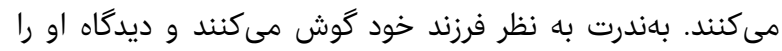

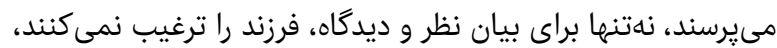

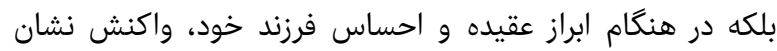

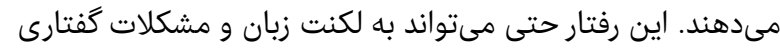

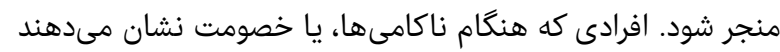

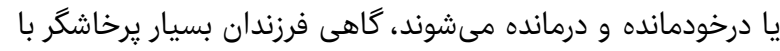

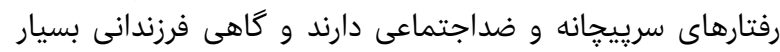

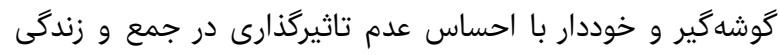

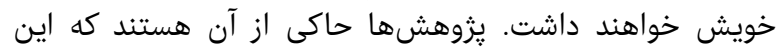

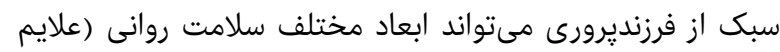

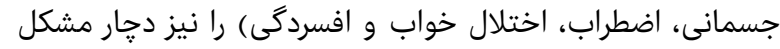

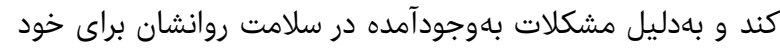
و ديكران ايجاد مخاطره نمايد [32-37].

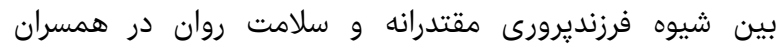

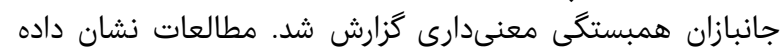

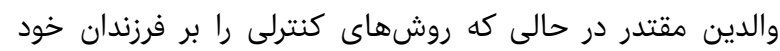

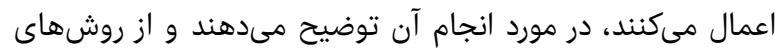

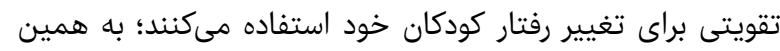

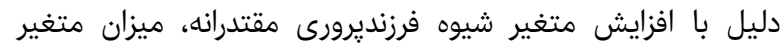

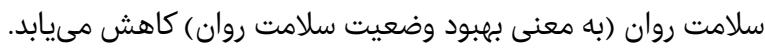

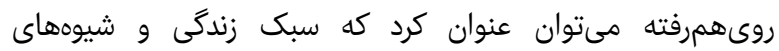

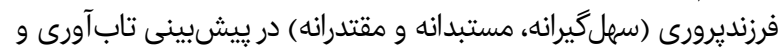

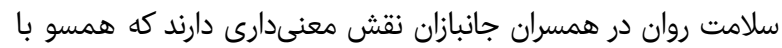

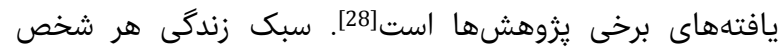

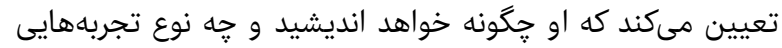

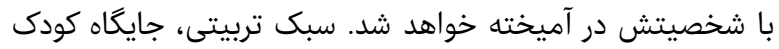

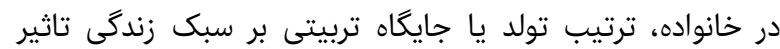

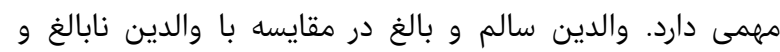

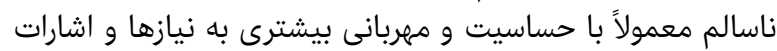

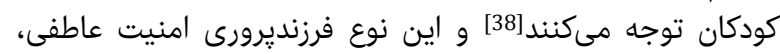

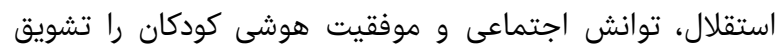

داده است كه آموزشهاى سلامت روانى مىتواند در بهبود كيفيت

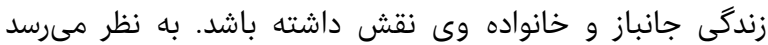

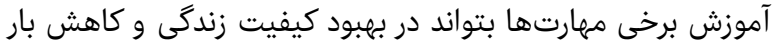

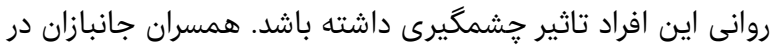

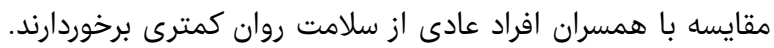

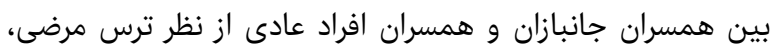

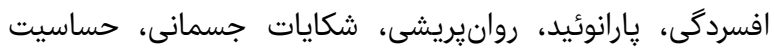

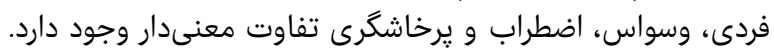

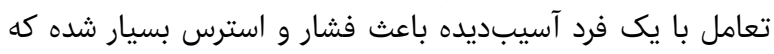

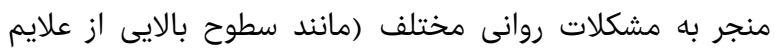

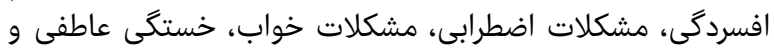

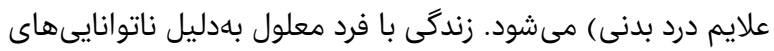

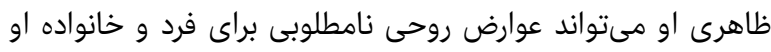

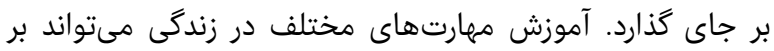

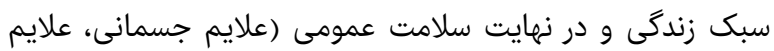

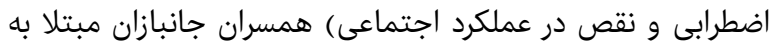

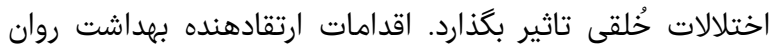

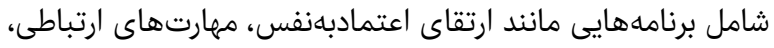

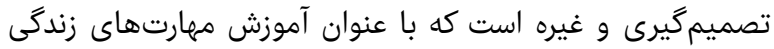

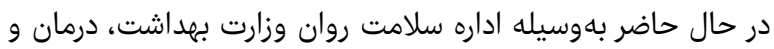
آموزش يزشكى، سازمان بهزيستى در حال انهار انجام است.

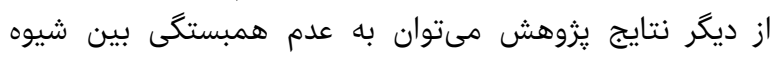

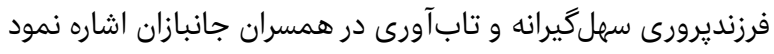

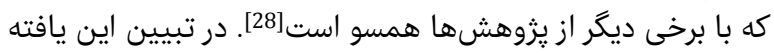

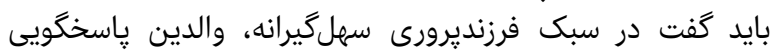

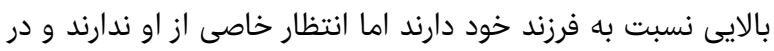

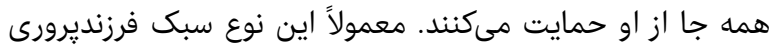

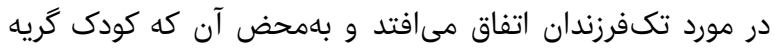

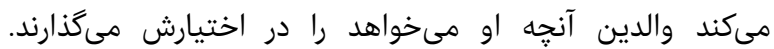

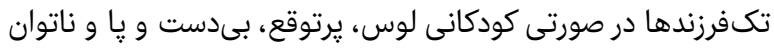

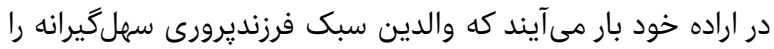

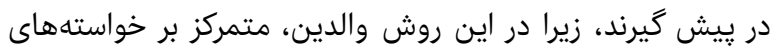

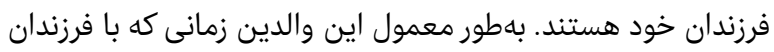

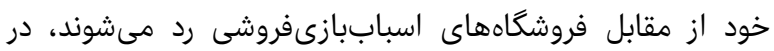

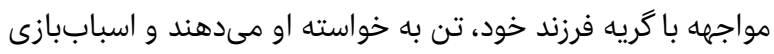

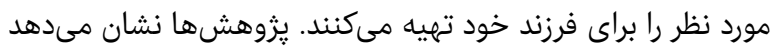

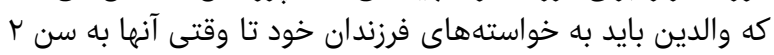

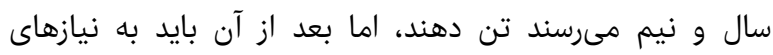

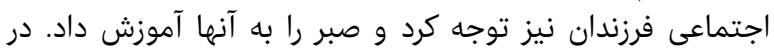

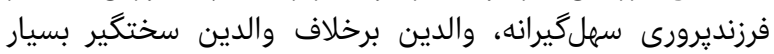

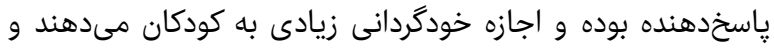

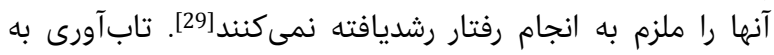

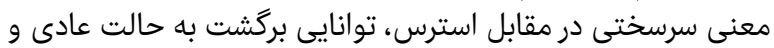

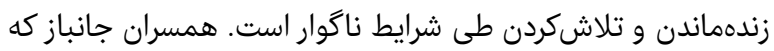

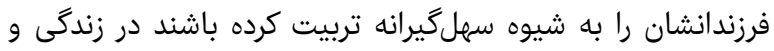

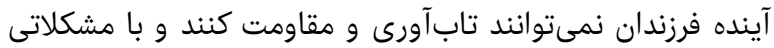
مواجه خواهند شد.

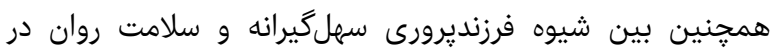

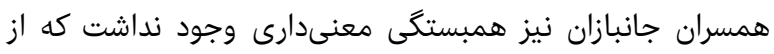

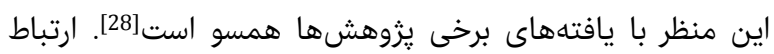

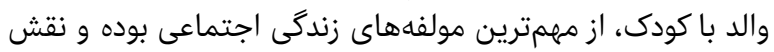

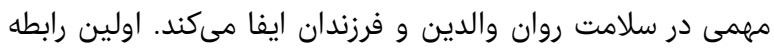


3- Haditabar H, Navabinezhad S, Ahghar GH. The effect of spiritual components training on quality of life of veterans' spouses working in adult schools in Tehran. Iran J War Public Health. 2012;4(3):1-11. [Persian]

4- Vafaei T, Khosravi S. comparison between mental health of devotee's spouses and normal person's spouses. Iran J War Public Health. 2009;1(4):9-13. [Persian]

5- Yamininia N. Investigation of personality traits of wives of spinal cord and spinal cord injury and spouses of non-injured individuals [Dissertaton].Tehran: Tarbiat Moallem University; 2001. [Persian]

6- Shoarinejad AA.Growth Psychology (1). Tehran: Payame Noor University; 2006. [Persian]

7- Akbarizadeh F, Bagheri F, Hatami HR, Hajivandi A. Relationship between spiritual intelligence, hardiness and general health among nurses. J Kermanshah Univ Med Sci. 2012;15(6):466-72. [Persian]

8- James Saduk B, Alcott Saduk V, Roez P. Kaplan \& Sadock's synopsis of psychiatry behavioral sciences. Rezaei F, translator. Volume 2. $2^{\text {nd }}$ Edition. Tehran: Arjmand; 2011 [Persian].

9- Javadi R, Parv D. The relationship between emotional intelligence and resilience in students at university of social welfare science and rehabilitation. J Res Addict. 2009;2(8):69-82. [Persian]

10- Fauci AJ, Bonciani M, Guerra R. Quality of life, vulnerability and resilience: a qualitative study of the tsunami impact on the affected population of Sri Lanka. Ann Ist Super Sanità. 2012;48(2):177-88.

11- Murthy RS, Lakhminarayana R. Mental health consequences of war: a brief review of research findings. World Psychiatry. 2006;5(1):25-30.

12- Ganzer CA. Veteran women: mental health-related consequences of military service. Am J Nurs. 2016;116(11):32-9.

13- MacDonell GV, Bhullar N, Thorsteinsson EB. Depression, anxiety, and stress in partners of Australian combat veterans and military personnel: a comparison with Australian population norms. Peer J. 2016;4:e2373.

14- Krešić Ćorića M, Klarića M, PetrovaB, Mihićb N. Psychological and behavioral problems in children of war veterans with post traumatic stress disorder. Eur J Psychiatry. 2016;30(3):219-30.

15- Quinn M. Psychosocial outcomes and predictors of distress among military spouses [Dissertaton]. Melbourne: Victoria University; 2017.

16- Devine DT. Levels of distress among women veterans attending a women's health specialty clinic in the VA healthcare system. [Dissertaton]. Tampa, Florida: University of South Florida; 2016.

17- Pemberton MR, Forman-Hoffman VL, Lipari RN, Ashley OS, Heller DC, Williams MR. Prevalence of past year substance use and mental illness by veteran status in a nationally representative sample. Center for Behavioral Health Statistics and Quality data review. 2016.

18- Ahmadi MS. Religious beliefs and lifestyle with quality of life of second grade high school students in Zanjan. Woman Fam Stud. 2015;8(3):8-20. [Persian]

19- Afzalnia MR. Family mental health. $1^{\text {st }}$ Edition. Tehran: Nasl-e noandish; 2007. [Persian]

20- Hasanzadeh R, Farzan S, Khodemlou A. Theories and application of psychological and personality tests. Tehran: Arasbaran; 2014. [Persian]

21- Farahini N, Afrouz G, Rasoulzadeh Tabatabaei SK. The relationship between parenting styles, shyness and

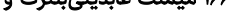

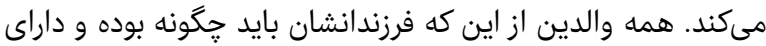

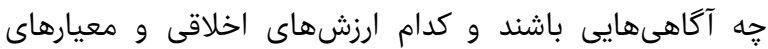

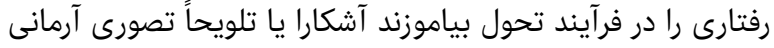

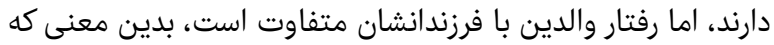

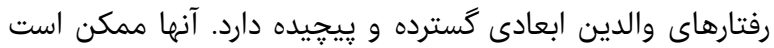

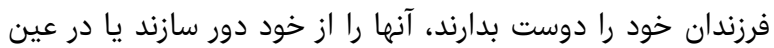

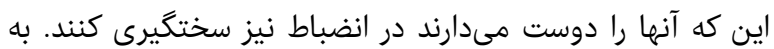

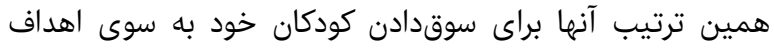

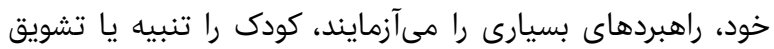

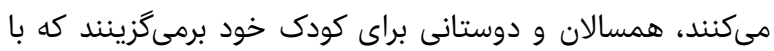

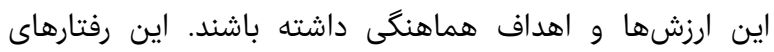

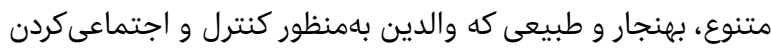

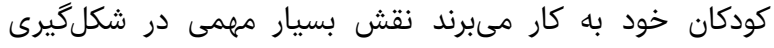

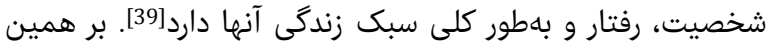

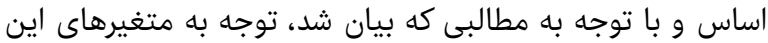
يزوهش ضرورى به نظر مى بـرسد.

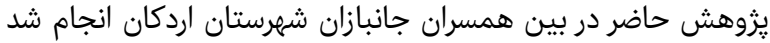

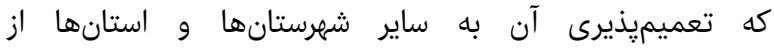

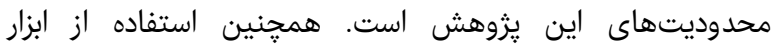

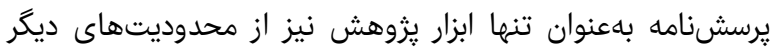

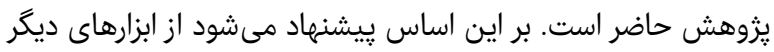

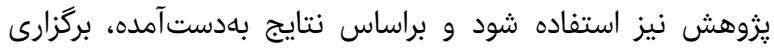

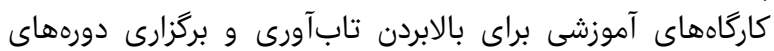

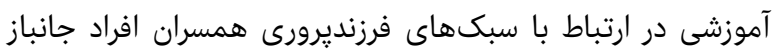
ي ي منشهاد مى شود. نتيجهگيرى سيك زندى و شيوههاى فرزنديرورى با تابآورى و سلامت روان در تشكر و قدردانى: نويسندگًان از كليه افرادى كه در انجام هر جها بهرتر يزوهش ياريخر بودند، قدردانى مى تئمايند.

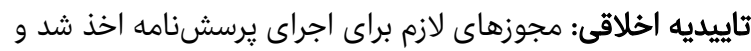

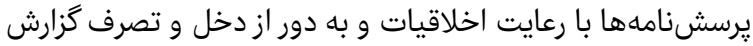
شده است. تعارض منافع: هيج گَنه تعارض منافعى در يزوهش حاضر وجود

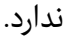

سهم نويسندگان: ميمنت عابدينى بلترك (نويسنده اول)، نگارنده

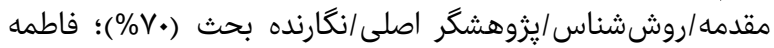

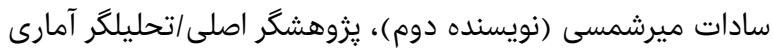

منابع مالى: هيج حمايت مالى توسط سازمانى صورت نكرفته و هزينهها توسط نويسندكان تامين مالين شده است.

منابع

1- Parandeh A, Sirti M, Khaghani zadeh M, Karimi zarji AA. The effect of training conflict resolution on quality of life's on spouses of war veterans post traumattic stress disorder. J Mil Med. 2006;8(1):45-51. [Persian]

2- Gholipour S, Nazari AA, Gholipour M. Directory services to veterans and their quality life (case study: Kermanshah). J Contemp Soc Res. 2018;6(11):149-75. [Persian] 
IFV همبستكى تابآورى و سلامت روان با سبك زندگى و سبكهاى فرزنديرورى همسران جانبازان Davoodi I, Marashi SA. The effectiveness of mindfulnessbased stress reduction on quality of life and resiliency of veterans spouses in Ahvaz. Psychol Methods Models. 2016;7(23):83-99. [Persian]

31- Seifi M. The effects of parenting styles on students' self-efficacy. Int J Hum Cult Stud. 2016;special Issus:2193-2200

32- Mishra P, Kiran UV. Parenting style and social anxiety among adolescents. Int J Appl Home Sci. 2018;5(1):11723.

33- Mousavi SE, Low WY, Hashim AH. The relationships between perceived parental rearnig style and anxiety symptoms in malaysisan adolescents: the mediating role of early maladaptive schemas. J Depress Anxiety. 2016;2(9):1-8.

34- Hanafi H, Mousa Thabet AA. The relationship between parenting styles and mental health problems among preschool children living in Gaza Strip. EC Psychol Psychiatry. 2017;7(7):347-57.

35- Zhong X, Wu D, Nie X, Xia J, Li M, Lei F, et al. Parenting style, resilience, and mental health of community-dwelling elderly adults in China. BMC Geriatr. 2016;16:135.

36- Xu J, Ni S, Ran M, Zhang C. The relationship between parenting styles and adolescents' social anxiety in migrant families: a study in Guangdong. China. Front Psychol. 2017;8:626.

37- Eman Shokry A, Hanna Hamdy, Rehab Fathy $\mathrm{AH}$, Marwa EL-Sh. Association between social phobia and parenting styles among secondary school students. Am J Nurs Sci. 2016;5(3):96-105.

38- Mahaptra S, Batul R. Psychosocial consequences of parenting. J Hum Soc Sci. 2016;21(2):10-7.

39- Sarwar S. Influence of parenting style on children's behaviour. J Educ Educ Dev. 2016;3(2):221-49. creativity in the gifted. J Sch Psychol. 2013;2(4):137-53. [Persian]

22- Bigdeli I, Najafi M, Rostami M. The relation of attachment styles, emotion regulation, and resilience to well-being among students of medical sciences. Iran J Med Educ. 2013;13(9):721-29. [Persian]

23- Compell-Sills L, Stein MB. Psychometric analysis and refinement of the Conner-Davidson Resilience scale (CDRISC): Validation of a 10 item measure of resilience. J Trauma Stress. 2007;20(6):1019- 28.

24- Keyhani M, Taghvaei D, Rajabi A, Amirpour B. Internal consistency and confirmatory factor analysis of the Connor-Davidson Resilience Scale (CD-RISC) among nursing female students. Iran J Med Educ. 2015;14(10):857-59. [Persian]

25- Taghavi SMR. Assessment of the validity and reliability of the general health questionnaire. J Psychology. 2001;5(4):381-98. [Persian]

26- Nazifi M, Mokarami HR, Akbaritabar AA, Faraji Koujerdi M, Tabrizi R, Rahi A. Reliability, validity and factor structure of the Persian translation of General Health Questionnire (GHQ-28) in Hospitals of Kerman University of Medical Sciences. J Fasa Univ Med Sci. 2014;3(4):336-42. [Persian]

27- Jafarzadeh N. Relationship between parenting and resilience styles with happiness of students. J Educ Psychol. 2015;11(37):67-81. [Persian]

28- Ghasemi M, Gholami A, Mehdizadeghan I. The effect of training Forgiveness on Resilience and Perceived stress in Wives of Veteran. Iran J War Public Health. 2017;9(4):177-83.

29- Tozandehjani $H$, Tavakolizadeh J, Lazian Z. The effect of parenting styles on self-efficacy and mental health of Students. Horizon Med Sci. 2011;17(2):56-64. [Persian] 30- Zarnaghash M, Mehrabizadeh M, Bashlideh K, 